\title{
Biofilm formation at the solid-liquid and air-liquid interfaces by Acinetobacter species
}

\author{
Sara Marti ${ }^{1 *}$, Jesús Rodríguez-Baño ${ }^{2}$, Manuella Catel-Ferreira ${ }^{1}$, Thierry Jouenne ${ }^{1}$, Jordi Vila ${ }^{3}$, Harald Seifert ${ }^{4 *}$, \\ Emmanuelle Dé ${ }^{1}$
}

\begin{abstract}
Background: The members of the genus Acinetobacter are Gram-negative cocobacilli that are frequently found in the environment but also in the hospital setting where they have been associated with outbreaks of nosocomial infections. Among them, Acinetobacter baumannii has emerged as the most common pathogenic species involved in hospital-acquired infections. One reason for this emergence may be its persistence in the hospital wards, in particular in the intensive care unit; this persistence could be partially explained by the capacity of these microorganisms to form biofilm. Therefore, our main objective was to study the prevalence of the two main types of biofilm formed by the most relevant Acinetobacter species, comparing biofilm formation between the different species.
\end{abstract}

Findings: Biofilm formation at the air-liquid and solid-liquid interfaces was investigated in different Acinetobacter spp. and it appeared to be generally more important at $25^{\circ} \mathrm{C}$ than at $37^{\circ} \mathrm{C}$. The biofilm formation at the solid-liquid interface by the members of the ACB-complex was at least 3 times higher than the other species (80-91\% versus 5-24\%). In addition, only the isolates belonging to this complex were able to form biofilm at the air-liquid interface; between $9 \%$ and $36 \%$ of the tested isolates formed this type of pellicle. Finally, within the ACB-complex, the biofilm formed at the air-liquid interface was almost 4 times higher for A. baumannii and Acinetobacter G13TU than for Acinetobacter G3 (36\%, 27\% \& 9\% respectively).

Conclusions: Overall, this study has shown the capacity of the Acinetobacter spp to form two different types of biofilm: solid-liquid and air-liquid interfaces. This ability was generally higher at $25^{\circ} \mathrm{C}$ which might contribute to their persistence in the inanimate hospital environment. Our work has also demonstrated for the first time the ability of the members of the ACB-complex to form biofilm at the air-liquid interface, a feature that was not observed in other Acinetobacter species.

\section{Findings}

The observation of natural habitats has shown that bacteria generally aggregate in biofilm structures to persist [1]. Biofilm is an association of microbial cells which are surrounded by a matrix of polysaccharide material; this structure is an optimal environment for genetic material exchange between the different microorganisms [2]. Biofilm formation has been linked to the survival of pathogenic bacteria in the hospital environment and it has

\footnotetext{
* Correspondence: saramarti2@yahoo.es; harald.seifert@uni-koeln.de 'Laboratory "Polymères, Biopolymères, Surfaces", University of Rouen, UMR 6270 \& FR 3038 CNRS, IFRMP23, Mont-Saint-Aignan, France

${ }^{4}$ Institute for Medical Microbiology, Immunology and Hygiene, University of Cologne, Cologne, Germany

Full list of author information is available at the end of the article
}

been connected to infections associated with indwelling medical devices. Indeed, biofilm bacterial communities confer a protection from environmental hazards $[1,3]$. The surface colonization can take place either at the solid-liquid interface (SLI-biofilm) or at the air-liquid interface (ALI-biofilm) where it forms a pellicle on the top of the liquid media as observed in other microorganisms such as Pseudomonas aeruginosa or Salmonella spp. $[4,5]$.

The members of the genus Acinetobacter are ubiquitous Gram-negative cocobacilli that are frequently found in the environment but also in the hospital setting where they have been associated with outbreaks of nosocomial infections [6]. The so-called Acinetobacter calcoaceticus - Acinetobacter baumannii (ACB) complex
C Biomed Central

(c) 2011 Martí et al; licensee BioMed Central Ltd. This is an Open Access article distributed under the terms of the Creative Commons Attribution License (http://creativecommons.org/licenses/by/2.0), which permits unrestricted use, distribution, and reproduction in any medium, provided the original work is properly cited. 
contains phenotypically closely related species, i.e. the clinically most important species, A. baumannii, Acinetobacter Genospecies 3 and Acinetobacter Genospecies $13 \mathrm{TU}$, also referred to as the $A$. baumanni group, and the environmental species $A$. calcoaceticus [7]. Although these species are very difficult to differentiate in the laboratory, their importance in the clinical environment is clearly different: A. baumannii and Acinetobacter Genospecies $13 \mathrm{TU}$ are responsible for most of the infections while Acinetobacter Genospecies 3 is less often associated with disease. On the other hand, A. calcoaceticus is mainly an environmental microorganism rarely involved in human infections [7]. Among them, A. baumannii has emerged as the most common pathogenic species involved in hospital-acquired infections [6,8-10]; this multiresistant opportunistic pathogen can survive on nutrient-limited surfaces for several days, even in dry conditions and in the harsh hospital environment [11]. One reason for this emergence may be its persistence in the hospital wards, in particular in the intensive care unit; this persistence could be partially explained by the capacity of these microorganisms to form biofilm. Therefore, our main objective was to study the prevalence of the two main types of biofilm formed by the most relevant Acinetobacter species. Overall, this study has shown the capacity of the members of the ACB-complex to form two different types of biofilm (SLI and ALI), and it revealed that biofilm formation increased at $25^{\circ} \mathrm{C}$, a condition that might contribute to their persistence in the hospital environment.

\section{Bacterial strains}

This study has investigated the ALI and SLI-biofilm formation in different Acinetobacter spp.: 64 clonally unrelated $A$. baumannii clinical isolates collected during the GEIH-Ab2000 project [12]; Acinetobacter johnsonii ( $\mathrm{n}=$ 34); Acinetobacter lwoffii ( $\mathrm{n}=26)$; Acinetobacter radioresistens ( $\mathrm{n}=20)$; Acinetobacter Genospecies $3(\mathrm{n}=46)$; Acinetobacter Genospecies 13TU ( $\mathrm{n}=60)$; A. calcoaceticus $(\mathrm{n}=10)$; Acinetobacter junii $(\mathrm{n}=5)$. The Acinetobacter spp. other than A. baumannii were mainly recovered from catheter-related bloodstream infections and from the skin of patients outside the ICU and of healthy controls [13-15]. Both biofilm analyses were carried out on all the isolates in parallel.

\section{SLI-Biofilm formation}

SLI-biofilm formation was performed in 96-well plates; biofilm formation was determined in Mueller Hinton broth (Oxoid, France) using an initial $\mathrm{OD}_{600}$ of 0.01 and incubated at $25^{\circ} \mathrm{C}$ or $37^{\circ} \mathrm{C}$ for $48 \mathrm{~h}$ without shaking. Two wells were left uninoculated and used as negative controls. After checking that all the isolates had grown at a similar rate, the culture media was removed by inversion and the wells were washed twice with distilled water. The biofilm was stained with $0.5 \%$ crystal violet $(\mathrm{w} / \mathrm{v})$ for 20 minutes at room temperature and the wells were washed again to remove the unbound crystal violet. Biofilm formation was finally quantified at $550 \mathrm{~nm}$ after solubilisation with $95 \%$ ethanol. The bacterial isolates were considered to be positive for SLI-biofilm formation when the readings obtained were at least 3 times greater than the negative control.

\section{ALI-Biofilm formation}

ALI-biofilm formation was performed in $5 \mathrm{ml}$ polystyrene tubes with a diameter of $12.8 \mathrm{~mm}$; biofilm formation was determined in Mueller Hinton broth (Oxoid, France) using an initial $\mathrm{OD}_{600}$ of 0.01 and incubated at $25^{\circ} \mathrm{C}$ or $37^{\circ} \mathrm{C}$ for $72 \mathrm{~h}$ without shaking. Positive ALIbiofilm samples were identified visually (Figure 1); the isolates were considered positive when a pellicle was covering the whole liquid surface.

\section{Data analysis}

All the experiments were performed in duplicate at two independent time-points. The percentages of isolates producing biofilm were compared by the chi squared test, or the Fisher exact test, as appropriate. A 2- tailed $\mathrm{P}$ value $<0.05$ was considered significant. The percentage of isolates of the various Acinetobacter spp. producing biofilm is illustrated in Table 1.

\section{Results and discussion}

As shown in Table 1 SLI-biofilm was more frequently produced at both $25^{\circ} \mathrm{C}$ and $37^{\circ} \mathrm{C}$ in Acinetobacter Genospecies 3, Acinetobacter Genospecies 13TU, and A. baumannii than in $A$. johnsonii, A. lwoffii, and A. radioresistens ( $\mathrm{p}<0.05$ for all comparisons). Thus,

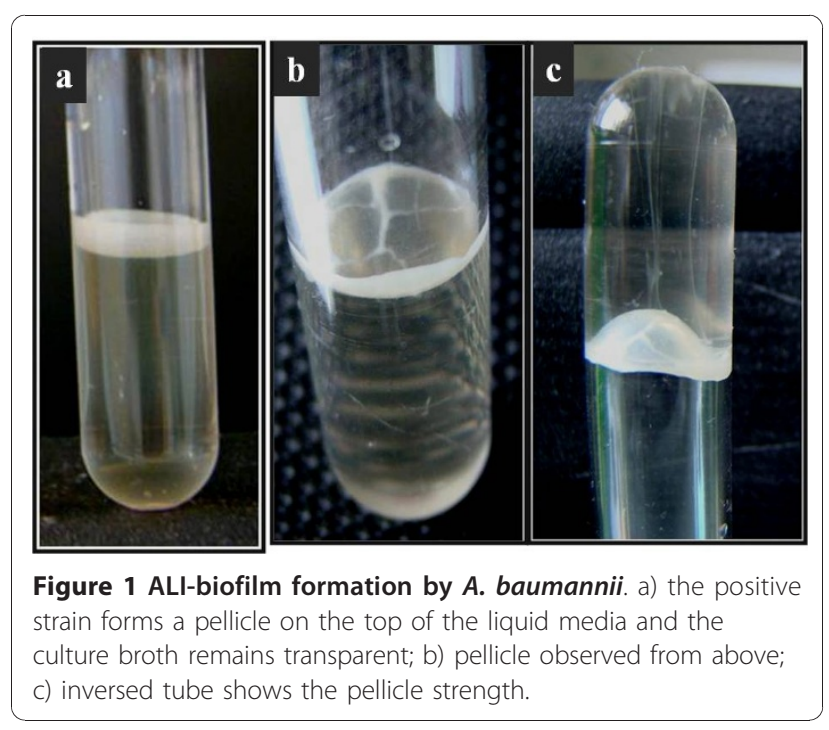


Table $1 \mathrm{SLI}$ and ALI-biofilm formation in Acinetobacter spp

\begin{tabular}{lllll}
\hline & \multicolumn{2}{l}{ ALI-Biofilm } & \multicolumn{2}{l}{ SLI-Biofilm } \\
\cline { 2 - 5 } & $\mathbf{2 5 ^ { \circ } \mathbf { C }}$ & $\mathbf{3 7 ^ { \circ } \mathbf { C }}$ & $\mathbf{2 5 ^ { \circ } \mathbf { C }}$ & $\mathbf{3 7 ^ { \circ } \mathbf { C }}$ \\
\hline A. baumannii $(\mathbf{n}=\mathbf{6 4})$ & $23(35.9)$ & $11(12.2)$ & $52(81.3)$ & $(16)^{\mathrm{a}}$ \\
Acinetobacter $\mathrm{G3}(\mathbf{n}=\mathbf{4 6})$ & $4(8.7)$ & $1(2.2)$ & $42(91)$ & $42(91)$ \\
Acinetobacter G13TU $(\mathbf{n}=\mathbf{6 0})$ & $16(26.7)$ & $9(15)$ & $48(80)$ & $34(56.7)$ \\
A. johnsonii $(\mathbf{n}=\mathbf{3 4})$ & 0 & 0 & $8(23.5)$ & $5(14.7)$ \\
A. Iwoffii $(\mathbf{n}=\mathbf{2 6})$ & 0 & 0 & $3(11.5)$ & $3(11.5)$ \\
A. radioresistens $(\mathbf{n}=\mathbf{2 0})$ & 0 & 0 & $1(5)$ & $3(15)$ \\
A. calcoaceticus $(\mathbf{n}=\mathbf{1 0})$ & $3(30)$ & 0 & $7(70)$ & $2(20)$ \\
A. junii $(\mathbf{n}=\mathbf{5})$ & $3(60)$ & $3(60)$ & $3(60)$ & $2(40)$ \\
\hline
\end{tabular}

Data are expressed as number (percentage) of isolates.

${ }^{\text {a }}$ The percentage of SLI-Biofilm formation in this collection of $A$. baumannii clinical isolates has already been published by Rodriguez-Baño et al (16); this study showed that $63 \%$ of the clinical isolates were forming SLI-biofilm at $37^{\circ} \mathrm{C}$.

these results might explain, at least in part, the marked persistence of the three former species in hospitals and their involvement in nosocomial infections. Moreover, in a previous study (using the same collection of A. baumannii clinical isolates), Rodriguez-Baño et al. [16] showed that $63 \%$ of $A$. baumannii clinical isolates formed SLI-biofilm at $37^{\circ} \mathrm{C}$. Based on these results, our study revealed that the rates of SLI-biofilm formation obtained for Acinetobacter Genospecies $13 \mathrm{TU}$ and $A$. baumannii were similar, as well as their variation related to the temperature (higher at $25^{\circ} \mathrm{C}$ than at $37^{\circ} \mathrm{C}$ ). Likewise, ALI-biofilm was significantly more frequent at both $25^{\circ} \mathrm{C}$ and $37^{\circ} \mathrm{C}$ in Acinetobacter Genospecies $13 \mathrm{TU}$ and $A$. baumannii than in $A$. johnsonii, A. lwoffii, $A$. radioresistens, and Acinetobacter Genospecies 3 ( $\mathrm{p}<$ 0.05). Overall, this similar behaviour in biofilm formation between $A$. baumannii and Acinetobacter G13TU is coherent with the fact that these two species are the most commonly found in the hospital.

Our results showed (see Table 1) that ALI-biofilm was mainly formed by the members of the ACB-complex. Although A. junii had some capacity to form this characteristic biofilm, the number of isolates studied was too small to draw conclusions. By contrast, as a member of the ACB-complex, $A$. calcoaceticus has shown a different pattern at $37^{\circ} \mathrm{C}$ with a complete absence of ALI-biofilm formation together with a reduced ability to form SLI-biofilm, both of which could be explained by the fact that this species' natural habitat is the environment where lower temperatures usually prevail.

Finally, as biofilm formation generally predominated at $25^{\circ} \mathrm{C}$ rather than $37^{\circ} \mathrm{C}$, this mechanism could explain the observed persistence of the members of the A. baumannii group in the inanimate hospital environment. Previous studies have already reported that the members of the $A$. baumannii group and especially $A$. baumannii survive desiccation better than other Acinetobacter spp., comparing the survival rate of $A$. baumannii to those obtained for Staphylococcus aureus [7,17]. In addition, in a recent study, Wisplinghoff et al showed that all disinfectants tested inhibited the growth of A. baumannii [18] which suggests that these microorganisms in their planktonic state are susceptible to most disinfectants. As already demonstrated for other bacterial species, extracellular polymeric substances from the biofilm matrix play an important role in the resistance and tolerance to dehydration [19], suggesting that biofilm formation contributes to the ability of $A$. baumannii, and possibly the other members of the $A$. baumannii group, to survive better in the hospital environment and to resist the action of disinfectants.

For A. baumannii, SLI-biofilm has already been described in several reports [20-22] and it has been linked to some device-associated infections [16]. Indeed, de Breij et al [21] have recently compared SLI-biofilm formation by the members of the ACB complex and concluded that there was no difference between clinically relevant and less-relevant Acinetobacter strains and species; in the same way, no temperature related differences were found for biofilm formation. Our results slightly differ from theirs possibly due to the higher number of strains and species analysed. They suggested a reduced biofilm formation for the Acinetobacter Genospecies 13TU although only 3 isolates had been studied; by contrast, after studying 60 isolates, our results clearly show that the behaviour for biofilm formation of this species is highly related to A. baumannii. On the other hand, although we have only studied $10 \mathrm{~A}$. calcoaceticus isolates, the results also indicate an association between biofilm and temperature in this non-pathogenic species, a tendency that can be observed in most of the analysed species. The comparison with other non-pathogenic species outside the ACB-complex has shown a relevant difference for biofilm formation that was even increased when looking at the ALI-biofilm formation, an ability that to our knowledge has never been described in clinical isolates of the Acinetobacter spp.

In summary, the members of the A. baumannii group have a higher ability to form SLI-and ALI-biofilm than other less clinically related species. Nevertheless, A. calcoaceticus, the environmental representative of the ACB-complex, showed a reduced biofilm formation at $37^{\circ} \mathrm{C}$ when compared to the other members of this complex. This feature could be connected to the higher colonization rate of patients by pathogenic Acinetobacter species (mainly $A$. baumannii and Acinetobacter Genospecie 13TU), and probably contributing to the increased risk of clinical infection.

\section{Consent}

Due to the observational and retrospective design of the study, the Ethic Committee of the participating centres waived the need for obtaining written informed consent. 


\section{List of abbreviations}

SLI: Solid-Liquid Interface; ALI: Air-Liquid Interface; ACB-Complex:

Acinetobacter calcoaceticus - Acinetobacter baumannii complex

\section{Acknowledgements}

The study was supported by the Centre National de la Recherche Scientifique (CNRS) and the Université de Rouen (France).

We thank the Grupo de Estudio de la Infección Hospitalaria (GEIH) from the Sociedad Española de Enfermedades Infecciosas y Microbiología Clínica (SEIMC) and Ministerio de Sanidad y Consumo, Instituto de Salud Carlos III FEDER, Spanish Network for the Research in Infectious Diseases (REIPI RD09/ 0008) for supporting this study. S.M has a post-doctoral fellowship from the region Haute Normandie.

\section{Author details}

'Laboratory "Polymères, Biopolymères, Surfaces", University of Rouen, UMR 6270 \& FR 3038 CNRS, IFRMP23, Mont-Saint-Aignan, France. ${ }^{2}$ Infectious Diseases and Clinical Microbiology Unit, Hospital Universitario Virgen Macarena, Seville, Spain. ${ }^{3}$ Department of Microbiology, Hospital Clinic, Barcelona, Spain. ${ }^{4}$ Institute for Medical Microbiology, Immunology and Hygiene, University of Cologne, Cologne, Germany.

\section{Authors' contributions}

SM carried out the biofilm studies, participated in the design of the study and drafted the manuscript; JRB performed the statistical analysis and helped to draft the manuscript; MCF helped to perform the biofilm studies; TJ participated in the design of the study; JV participated in the design of the study and helped to draft the manuscript; HS participated in the design of the study and helped to draft the manuscript; ED participated in the design and coordination of the study and helped to draft the manuscript. All authors have read and approved the final manuscript.

\section{Competing interests}

The authors declare that they have no competing interests.

Received: 7 September 2010 Accepted: 11 January 2011 Published: 11 January 2011

\section{References}

1. Davey ME, OToole GA: Microbial biofilms: from ecology to molecular genetics. Microbiol Mol Biol Rev 2000, 64:847-867.

2. Donlan RM: Microbial life on surfaces. Emerg Infect Dis 2002, 8:881-890.

3. Branda SS, Vik S, Friedman L, Kolter R: Biofilms: the matrix revisited. Trends Microbiol 2005, 13:20-26.

4. Friedman L, Kolter R: Genes involved in matrix formation in Pseudomonas aeruginosa PA14 biofilms. Mol Microbiol 2004, 51:675-690.

5. Solano C, Garcia B, Valle J, Berasain C, Ghigo JM, Gamazo C, Lasa I: Genetic analysis of Salmonella enteritidis biofilm formation: critical role of cellulose. Mol Microbiol 2002, 43:793-808.

6. Bergogne-Bérézin E, Towner KJ: Acinetobacter spp. as nosocomial pathogens: microbiological, clinical and epidemiological features. Clin Microbiol Rev 1996, 8:148-165.

7. Peleg AY, Seifert H, Paterson DL: Acinetobacter baumannii: emergence of a successful pathogen. Clin Microbiol Rev 2008, 21:538-582.

8. Dijkshoorn L, Nemec A, Seifert H: An increasing threat in hospitals: multidrug-resistant Acinetobacter baumannii. Nature Rev Microbiol 2007, 5:939-951.

9. Joly-Guillou M: Clinical impact and pathogenicity of Acinetobacter. Clin Microbiol Infect 2005, 11:868-873.

10. Wisplinghoff H, Hippler C, Bartual SG, Haefs C, Stefanik D, Higgins PG, Seifert H: Molecular epidemiology of clinical Acinetobacter baumannii and Acinetobacter genomic species 13TU isolates using a multilocus sequencing typing scheme. Clin Microbiol Infect 2008, 14:708-715.

11. Towner KJ: The genus Acinetobacter. Prokaryotes 2006, 6:746-758.

12. Rodriguez-Bano J, Cisneros JM, Fernandez-Cuenca F, Ribera A, Vila J, Pascual A, Martinez-Martinez L, Bou G, Pachon J: Clinical features and epidemiology of Acinetobacter baumannii colonization and infection in Spanish hospitals. Infect Control Hosp Epidemiol 2004, 25:819-824.

13. Seifert $H$, Strate A, Schulze A, Pulverer G: Vascular catheter-related bloodstream infection due to Acinetobacter johnsonii (formerly
Acinetobacter calcoaceticus var. Iwoffi): report of 13 cases. Clin Infect Dis 1993, 17:632-636.

14. Seifert H, Strate A, Schulze A, Pulverer G: Bacteremia due to Acinetobacter species other than Acinetobacter baumannii. Infection 1994, 22:379-385.

15. Seifert H, Dijkshoorn L, Gerner-Smidt P, Pelzer N, Tjernberg I, Vaneechoutte M: Distribution of Acinetobacter species on human skin: comparison of phenotypic and genotypic identification methods. J Clin Microbiol 1997, 35:2819-2825.

16. Rodriguez-Baño J, Marti S, Soto S, Fernandez-Cuenca F, Cisneros JM, Pachon J, Pascual A, Martinez-Martinez L, McQueary C, Actis LA, et al: Clin Microbiol Infect 2008, 14:276-278.

17. Jawad A, Seifert H, Snelling AM, Heritage J, Hawkey PM: Survival of Acinetobacter baumannii on dry surfaces: comparison of outbreak and sporadic isolates. J Clin Microbiol 1998, 36:1938-1941.

18. Wisplinghoff H, Schmitt R, Wohrmann A, Stefanik D, Seifert H: Resistance to disinfectants in epidemiologically defined clinical isolates of Acinetobacter baumannii. J Hosp Infect 2007, 66:174-181.

19. Stoodley P, Sauer K, Davies DG, Costerton JW: Biofilms as complex differentiated communities. Annu Rev Microbiol 2002, 56:187-209.

20. de Breij A, Gaddy J, van der Meer J, Koning R, Koster A, van den Broek P, Actis L, Nibbering P, Dijkshoorn L: CsuA/BABCDE-dependent pili are not involved in the adherence of Acinetobacter baumannii ATCC19606(T) to human airway epithelial cells and their inflammatory response. Res Microbiol 2009, 160:213-218.

21. de Breij A, Dijkshoorn L, Lagendijk E, van der Meer J, Koster A, Bloemberg G, Wolterbeek R, van den Broek P, Nibbering P: Do biofilm formation and interactions with human cells explain the clinical success of Acinetobacter baumannii? PLOS One 2010, 5:e10732.

22. Tomaras AP, Dorsey CW, Edelmann RE, Actis LA: Attachment to and biofilm formation on abiotic surfaces by Acinetobacter baumannii: involvement of a novel chaperone-usher pili assembly system. Microbiology 2003, 149:3473-3484.

\section{doi:10.1186/1756-0500-4-5}

Cite this article as: Martí et al:: Biofilm formation at the solid-liquid and air-liquid interfaces by Acinetobacter species. BMC Research Notes 2011 4:5.

\section{Submit your next manuscript to BioMed Central and take full advantage of:}

- Convenient online submission

- Thorough peer review

- No space constraints or color figure charges

- Immediate publication on acceptance

- Inclusion in PubMed, CAS, Scopus and Google Scholar

- Research which is freely available for redistribution

Submit your manuscript a www.biomedcentral.com/submit
Ciomed Central 